\title{
Le déroulement de la fraie des principaux poissons lacustres
}

\section{Gillet}

I.N.R.A. Station d'hydrobiologie lacustre 75, Avenue de Corzent - BP 11 F 74203 Thonon-lesbains

Résumé - La connaissance des conditions dans lesquelles se déroule la fraie des poissons lacustres constitue le point de départ pour l'aménagement et la protection des frayères naturelles et pour le choix des espèces susceptibles d'être acclimatées dans les lacs et les réservoirs.

Parmi les principaux facteurs influençant le succès de la fraie, les points suivants sont abordés :

- date de fraie,

- régimes thermiques permettant le déclenchement de la fraie et le bon déroulement du développement embryonnaire, ainsi que la gamme de degrés-jours de cette étape;

- substrats de ponte: la plasticité ou les exigences des espèces vis-à-vis des matériaux constituant les frayères sont discutés;

- profondeur des frayères pour chaque espèce et plasticité de certaines espèces vis-à-vis de ce paramètre.

Ces informations concernent les espèces suivantes:

- salmonidés (corégone et omble chevalier),

- percidés ( perche fluviatilis et sandre),

- brochet,

- cyprinidés (plus particulièrement le gardon).

L'existence de races géographiques et de sous-populations présentant des habitudes de fraie très différentes vis-à-vis de l'environnement (date de ponte, type de substrat ou profondeur), ainsi que l'existence de choming" pour les aires de fraie, apparaissent comme des phénomènes assez répandus chez les poissons lacustres.

Summary - Knowledge of the conditions in which the spawning of lake fishes occurs is the departure point in the management and protection of natural spawning grounds and in the choice of species which can be acclimated to lakes and reservoirs.

Some of the main environmental factors affecting successful spawning have been discussed :

- spawning date, 
- temperature regimes permitting the onset of spawning and good embryonic development as well as the degrees-day range for this stage, grounds,

- spawning substrates: species flexibility of demands in the matter of spawning

- depth of the spawning grounds for each species and the flexibility of some species in this regard.

These data concern the following species :

- salmonids (coregone and charr),

- percides (river perch, pike perch),

- pike

- cyprinids particularly roach.

The existence of geographic breeds and sub-populations having very different spawning habits in relation with the environment (spawning date, type of substrate or depth), as well as a sense of "homing" in regard to the spawning grounds, seem to be rather common phenomena among lake fishes.

Mots-cles: Poissons - Lacs - Réservoirs - Reproduction - Frayères.

\section{INTRODUCTION}

Les fluctuations du niveau d'eau dans les retenues ont un retentissement considérable sur le succès de la reproduction des poissons (MARTIN et al., 1981). En effet, la fraie d'un grand nombre d'espèces se déroule dans la ceinture de végétation de la zone littorale. Cette végétation est souvent dégradée, parfois même inexistante dans certains réservoirs à niveau variable.

La protection des frayères naturelles ou l'aménagement de frayères artificielles est un travail délicat car chaque espèce a des exigences précises en ce qui concerne le choix du substrat et la profondeur de sa frayère. De plus, sous nos latitudes, les périodes de reproduction des principales espèces lacustres se succèdent depuis la fin de l'automne jusqu'au début de l'été. II est donc nécessaire de bien connattre les exi- gences écologiques de la fraie de chaque espèce de poissons, pour aménager ou protéger efficacement ses frayères.

\section{I - LA FRAIE DES CYPRINIDES}

\section{A - L'exemple du gardon}

1) Le contrôle de la période de reproduction par les variations de la température

Les dates citées dans la littérature pour la fraie du gardon recouvrent les mois d'avril, mai et juin. BRAY (1971) signale même la présence d'oeufs de gardons dans le canal de rejet d'une centrale thermique, à $20^{\circ} \mathrm{C}$, le 20 mars. Nos dernières observations confirment l'existence d'un seuil de $20^{\circ} \mathrm{C}$ environ, pour l'initiation de la fraie du gardon. Le contrôle de la date de fraie, par un seuil de température, 
peut s'expliquer ainsi: l'élévation de la température stimule la secrétion d'hormone gonadotrope hypophysaire chez les Cyprinidés (GILLET et al., 1977 : BRETON et al., 1980). Chez une espèce voisine du gardon, Carassius auratus, STACEY et al. (1979) ont montré qu'un choc thermique provoque une forte décharge d'hormone gonatotrope chez les femelles qui vont frayer le lendemain.

L'amplitude des fluctuations journalières de température peut excéder $5^{\circ} \mathrm{C}$ à l'époque de la fraie du gardon. Ces variations expliquent pourquoi la ponte peut se dérouler à des températures nettement inférieures à $20^{\circ} \mathrm{C}$ : $14^{\circ} \mathrm{C}$ MILLS (1981), $17^{\circ} \mathrm{C}$ EASTON et al. (1980). Les populations les plus nordiques pourraient se reproduire à des températures plus basses. Les auteurs scandinaves citent des températures de 10 à $12^{\circ} \mathrm{C}$ pour la fraie du gardon (L'ABEE-LUND et al., 1985). La fraie de l'ensemble de la population dure environ trois jours dans le Léman, lorsque les conditions sont favorables. Le mauvais temps peut interrompre temporairement ou définitivement la fraie.

\section{2) L'emplacement et la composition des zones de frayères}

La plupart des frayères de gardon décrites dans la littérature sont des zones peu profondes, recouvertes de végétaux aquatiques. Les oeufs de cette espèce ont été observés sur un grand nombre de substrats (tableau 1). La structure des végétaux utilisés comme frayère a plus d'importance que leur nature. Ces plantes constituent un réseau très dense où les oeufs du gardon se fixent facilement. Leur adhérence est facilitée par une couche de mucus sur la partie externe de l'oeuf.

II existe aussi des populations de gardons qui ont l'habitude de déposer leurs oeufs sur les galets et les en-

Tableau 1 : Composition et profondeur des frayères naturelles du gardon Rutilus rutilus $\mathrm{L}$.

\begin{tabular}{|c|c|c|c|}
\hline Auteurs & Mlleu d'étude & Substrat de frayere & Profondeur \\
\hline \multicolumn{4}{|l|}{ A - Populations phytophiles } \\
\hline $\begin{array}{l}\text { MILLS, 19\&1 } \\
\text { PECZALSKA, } 1968 \\
\text { WILKOHSKA et ZUROHSKA, } 1967 \\
\text { GILLET }\end{array}$ & $\begin{array}{l}\text { River frone } \\
\text { Canaux } \\
\text { Lacs de Mazurie } \\
\text { Balastiere }\end{array}$ & $\begin{array}{l}\text { Fontinalis } \\
\text { Plantes aquatiques } \\
\text { 1) bare adrécoqeuse } \\
\text { 2) prairie inondee } \\
\text { 3) type interádidire } \\
\text { Racines de saule }\end{array}$ & $\begin{array}{l}0 \text { d } 9 \text { : } \\
\text { quelqups rentinetres } \\
\text { de la surface } \\
0,1 \text { d } 1,5= \\
0,1 \text { d } 0,5= \\
0,2 \text { d } 1,0 \text { : } \\
0,1 \text { d } 0,5=\end{array}$ \\
\hline \multicolumn{4}{|l|}{ B-Populations lithophiles } \\
\hline HOLCIK et HRUSKA, $1964-65$ & $\begin{array}{l}\text { Lac Klicaua } \\
\text { Lac Léman }\end{array}$ & $\begin{array}{l}\text { Rochers et oalets } \\
\text { Rochers }\end{array}$ & $\begin{array}{l}0 \text { d } 1,5 \text { : } \\
\text { profondeur moyenne: } \\
0,4: \text { par telaps chaud } \\
0,1 \text { par teaps froid } \\
0,08 \text { d } 1,5 \text { : }\end{array}$ \\
\hline
\end{tabular}


rochements (tableau 1b) Ces pois- leurs oeufs sur des substrats artifisons ont une fraie de type lithophile ciels lorsque ceux-ci ont une texture tandis que les premiers sont qualifiés voisine des substrats naturels. Les de phytophiles (BALON, 1975). Tous oeufs du gardon sont toujours très ces poissons acceptent de déposer proches de la surface. Les profon-

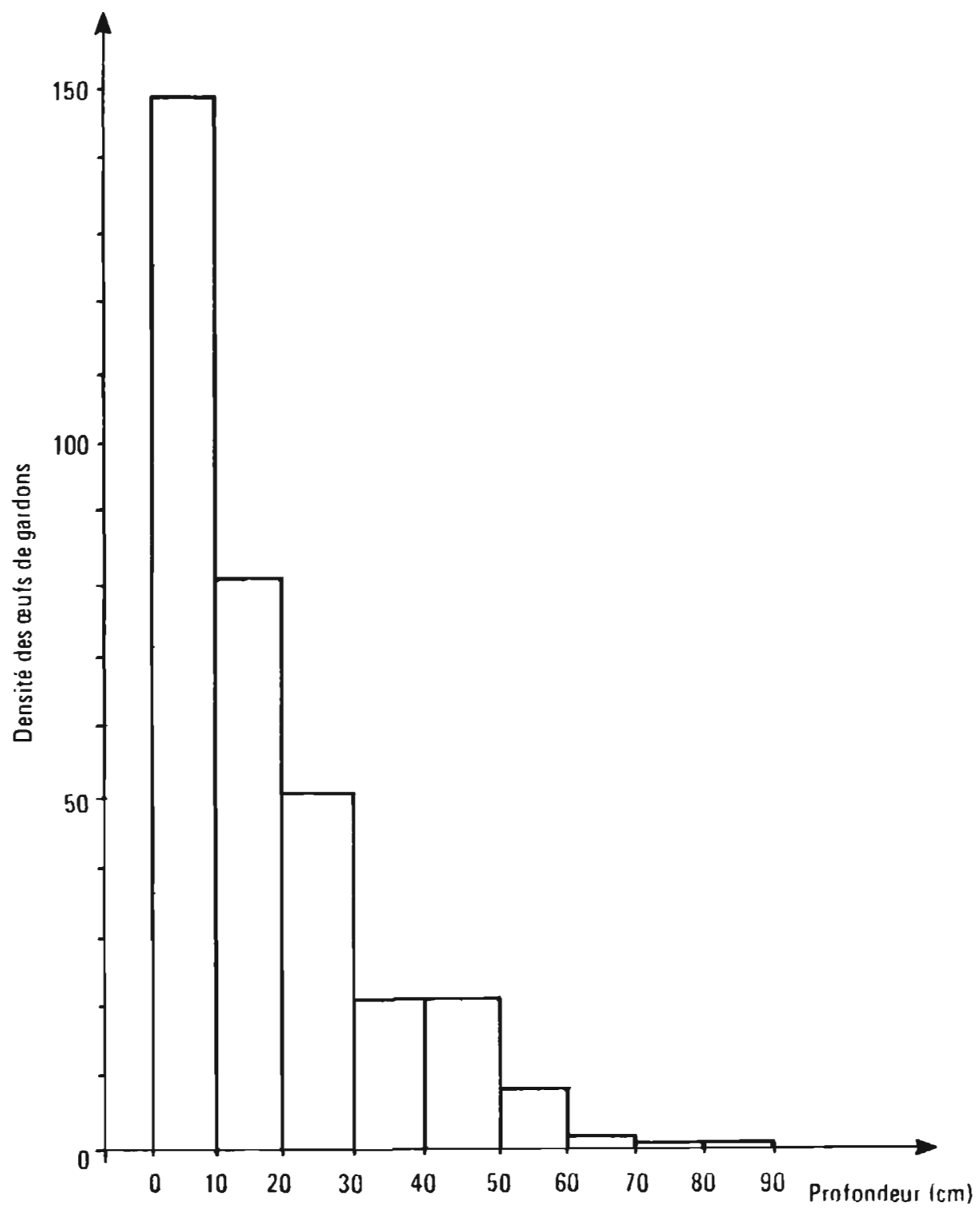

Figure 1 : Répartition des oeufs de gardons sur de la fontinale d'après MILLS (1981). 
deurs citées varient de quelques $\mathrm{cm}$ à quelques mètres. C'est surtout dans les premiers centimètres que se trouve concentrée la majorité des oeufs (tableau 1 et figures 1 et 2 ). Lorsque les conditions climatiques sont moins favorables, les oeufs peuvent être déposés à des profondeurs légèrement supérieures (tableau 1).

Dans le lac Léman, nous avons observé en 1986 et 1987, des dépôts d'oeufs par $4 \mathrm{~m}$ de fond, après une dégradation des conditions météorologiques. La stratification thermique de la colonne d'eau sur les premiers mètres est détruite par le vent et le refroidissement de l'air, ce qui pourrait expliquer pourquoi les poissons ne sont plus attirés par la couche d'eau chaude en surface.

La densité des oeufs sur les frayères est généralement supérieure à 10000 unités $/ \mathrm{m}^{2}$ (tableau 2) Les aires de fraie ont une surface limitée, les plus petites zones de frayères des lacs de Mazurie ont une surface de quelques dizaines de $\mathrm{m}^{2}$ (WILKOMSKA et ZUROMSKA, 1967).

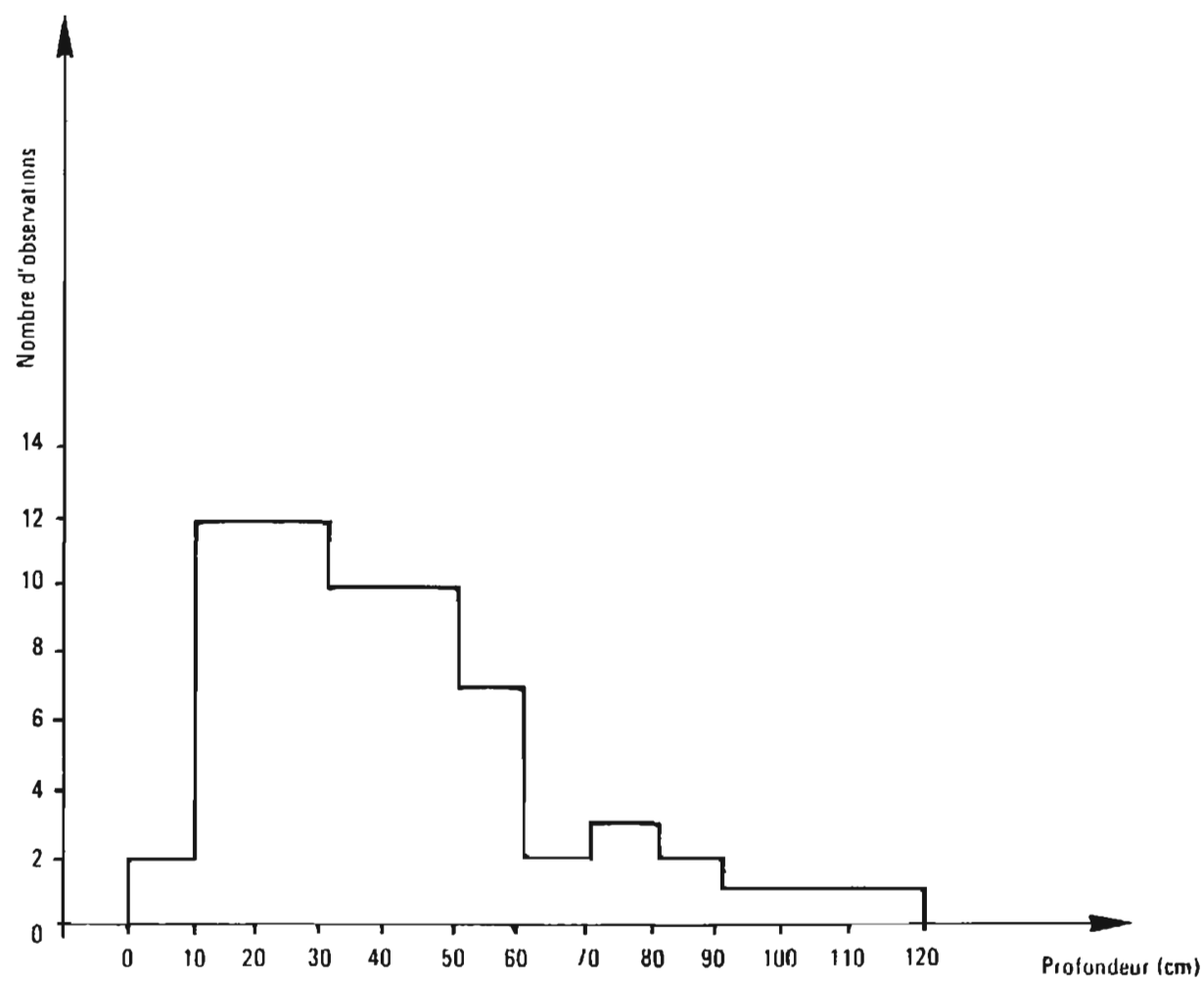

Figure 2 : Répartition des oeufs de gardons sur des rochers. Léman. 
Tableau 2 : Pourcentage de fécondation et densité des oeufs de gardons sur les frayères.

\begin{tabular}{|c|c|c|c|}
\hline Sites & Substrat de la frayere & $\begin{array}{l}\text { Taux of } \\
\text { tecondation }\end{array}$ & Densité des oeufs \\
\hline $\begin{array}{l}\text { Rigpe strume } \\
\text { Lacs se Mazurie }\end{array}$ & $\begin{array}{l}\text { Fortina! is } \\
\text { Plantes dquatiques }\end{array}$ & 95 वे $95:$ & $\begin{array}{l}2,05 / 5 \text { c (KILLS, 1981) } \\
2,7 \text { d } 40 / \text { CZ (ZYROHSKA, 1967) }\end{array}$ \\
\hline $\begin{array}{l}\text { LPBan 1997 } \\
" \\
\text { Baldatiere 14GZ }\end{array}$ & $\begin{array}{l}\text { Aads entre les rochers } \\
\text { Branches o'kolced } \\
\text { Ireillis en ol astique } \\
\text { Pacines de saule }\end{array}$ & $\begin{array}{l}1 \\
1,99: \\
198:\end{array}$ & $\begin{array}{ll}167 \pm 16 / \mathrm{C} 3 & \text { J Gillet } \\
500.000 \mathrm{pdr} \text { fagot } & 1,1989 \\
200.000 / C \backsim 2 & 1 \\
2,87 \pm 0,2 / \mathrm{c} & \text { J }\end{array}$ \\
\hline
\end{tabular}

3) L'influence des facteurs externes au cours de la période d'incubation

Le taux de fertilisation des oeufs est généralement très élevé dans les conditions naturelles. II est en général supérieur à $90 \%$ (tableau 2). L'oeuf du gardon supporte des températures de $12{ }^{\circ} \mathrm{C}$ à $24^{\circ} \mathrm{C}$ (GULIDOU et POPOVA, 1982). En raison de l'étendue de la gamme des températures favorables au déroulement du développement embryonnaire, les refroidissements provoquent rarement des mortalités importantes pendant l'incubation. Les températures croissantes constituent le meilleur régime thermique pour cette phase car de telles conditions permettent dans un premier temps le passage des réserves vitellines vers les cellules embryonnaires et par la suite l'utilisation de ces réserves pour les synthèses embryonnaires (REZNITCHENKO, 1971-1976). La durée de l'incubation varie entre 5 et 10 jours suivant la température de l'eau. Cette période est parfois mesurée en degrés-jours mais ce paramètre reste très approximatif car son utilisation es fondée sur une variation hyperbolique de la durée du développement embryonnaire en fonction de la température. Cette variation serait en réalité de type exponentielle chez les cyprinidés (MILLS, 1980). Les oeufs de gardons sont très sensibles à la pollution par les pluies acides pendant les premiers stades de l'embryogénèse (GILLET et ROUBAUD, 1986).

Les deux principaux facteurs de mortalité embryonnaire du gardon sur les frayères sont les vagues qui arrachent un grand nombre d'oeufs à leurs substrats et la prédation par les poissons ou les invertébrés. Ces deux facteurs cumulés représentent un pourcentage de perte de 95 à $99 \%$ pour les oeufs de gardons dans les lacs de Mazurie (ZUROMSKA, 1967). Dans le lac Léman nous n'observons pas de mortalité importante des oeuts de gardons. La densité des oeufs sur les frayères ne varie pas au cours de l'incubation (GILLET, 1989).

\section{4) L'existence d'une mémorisation des aires de fraie}

Dans plusieurs lacs, les populations de gardons retournent chaque année frayer sur les mêmes zones, bien que celles-ci ne représentent partois qu'un 
faible pourcentage de l'ensemble des frayères potentielles du plan d'eau. Le phénomène a été décrit dans le lac Sniardwy en Mazurie. Ce lac, d'une superficie de 110000 ha possède une baie peu profonde, de 680 ha, reliée au lac par un canal (figure 3). La totalité des gardons du lac viennent se reproduire chaque année dans cette baie, bien qu'il existe d'autres frayères potentielles dans le plan d'eau. Des expériences de marquage ont confirmé que les gardons qui frayent dans la baie proviennent de tous les points du lac (WILKOMSKA, 1977). Dans le lac Tjeukemeer, aux Pays-Bas, GOLDSPINK (1977) a constaté que les poissons qui se reproduisent sur deux aires de fraie distinctes retournent préférentiellement sur la frayère où ils ont été capturés lorsqu'on les relâche dans d'autres parties du lac. Dans le lac Orava, en Slovaquie, il existe une sous-population de gardons phytophiles qui se reproduit sur les herbiers de la zone littorale et une sous-population lithophile qui remonte le cours d'une rivière pour trayer parmi les galets (HOLCIK et HRUSKA, 1966). Dans un lac du sud de la Norvège (L'ABBELUND et al., 1985) ont décrit un phénomène de homing comparable à celui observé par GOLDSPINK (1977). II semble toutefois que ce homing soit moins strict que chez le saumon. Le pourcentage des échanges entre deux zones de frayères est de l'ordre de $20 \%$.

\section{B - La fraie des cyprinidés à pontes fractionnées}

1) Le contrôle de la date et de la durée de la période de fraie par les variations de la température.

La femelle du gardon dépose l'ensemble de ses oeufs en une seule fois. Chez la plupart des autres espèces de Cyprinidés, les femelles
A Lac Sniardwy $\cdot 11010$ ha
B Lac Luknajno $680 \mathrm{ha}(3 \mathrm{~m})$
- Point de recapture de gardons venus frayer dans le lac Luknajno

Tous les gardons du lac Sniardwy viennent frayer dans le lac Luknajno. Les hachurés corresp ondent à des trayères potentielles inutilišes par les gardons.

Figure 3 : Homing chez le gardon (d'après WILKONSKA, 1967).

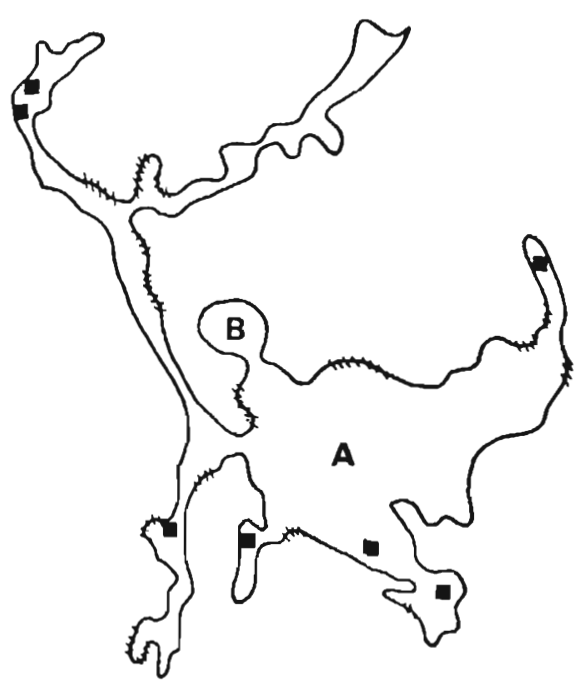


ovulent partiellement. La reproduction de chaque femelle comprend plusieurs pontes successives qui sont séparées par des intervalles de quelques jours ou quelques semaines. La tanche, la brème commune, la brème bordelière, la carpe, le rotengle et l'ablette sont des espèces à pontes fractionnées L'influence de la tempéature sur la date et le rythme de fraies a été étudiée chez la tanche par HOROSZEWICZ et al. (1981). Le cycle reproducteur de cette espèce a été observé dans des étangs réchauftés de plus $3^{\circ} \mathrm{C}$ et plus $6^{\circ} \mathrm{C}$ par les effluents d'une centrale thermique. Dans tous les cas, la première ponte survient lorsque la température de leau atteint environ $22{ }^{\circ} \mathrm{C}$. La fraie commence le 20 mai dans l'étang le plus chaud et un mois plus tard dans l'étang témoin qui a une température inférieure de $6{ }^{\circ} \mathrm{C}$. Dans tous les étangs la fraie se poursuit jusqu'au début du mois d'août. Les femelles de l'étang le plus chaud ont frayé en moyenne 6 fois de suite tandis que celles du milieu le plus froid n'ont frayé que 3 fois. Diverses observations réalisées dans des milieux naturels permettent d'étendre ce type de résultats à la majorité des cyprinidés à pontes fractionnées. Le rotengle et l'ablette frayent un plus grand nombre de fois dans les zones réchauffées vers $26{ }^{\circ} \mathrm{C}$ du réservoir Ivankov que dans les parties plus froides (EFIMOVA, 1977). Dans les régions les plus septentionales de leur aire géographique, certaines espèces comme la brème ne frayent plus qu'une seule fois. Lorsque la température de l'eau atteint au cours de la période de reproduction des valeurs très élevées, $28^{\circ} \mathrm{C}$ ou plus, la fraie de la brème, du rotengle et de l'ablette sont inhibées (STATOVA, 1973) ainsi que celle de la tanche (HOROSCZEWICZ et al., 1981). Nos observations sur la fraie de la tanche dans le lac Léman, confirment les résultats publiés par (HOROSCZEWICZ et al., 1981). Les poissons commencent à frayer lorsque la température de l'eau, en surface, dépasse $22{ }^{\circ} \mathrm{C}$, ce qui se produit entre la fin du mois de mai et la fin du mois de juin suivant les conditions météorologiques. La fraie s'arrête lorsque la température décroit puis reprend chaque fois que l'eau atteint plus de $22^{\circ} \mathrm{C}$. La fraie s'arrête définitivement au mois d'août, quelle que soit la date à laquelle elle avait commencé. Dans le lac Léman et le lac du Bourget, l'ablette commence à frayer en même temps que la tanche, mais la fraie se termine un mois plus tôt.

\section{2) La structure et la composition des zones de frayères}

L'ablette fraye de préférence sur les graviers tandis que la tanche, la brème, le rotengle et la carpe déposent leurs oeufs sur les végétaux aquatiques. KENNEDY a décrit les frayères de la brème (1968) et celles du rotengle (1974) en Irlande. Le rotengle fraye à partir de mai, jusqu'en juiliet sur toutes sortes de plantes aquatiques : Renoncule, Callitriche, Carex, Prèle, Fontinale, Phragmite, Hippuris, etc...) dans une gamme de profondeur allant de 0,15 à $1 \mathrm{~m}$, parfois dans les mêmes sites que la tanche. La brème commune commence à frayer une ou deux se- 
maines avant le rotengle et la tanche, sur des sites généralement plus profonds que ces dernières espèces (de 0,5 à $3 \mathrm{~m}$ ). II existe même une observation sur la fraie de la brème à plus de $5 \mathrm{~m}$ (SHESTOPALOVA 1978. Les oeufs de la brème sont déposés sur les mêmes plantes que les oeufs du rotengle. Les pontes les plus profondes sont déposées sur les Charas et les Potamogetons. Dans certains lacs, il existe chez l'ablette et la brème, des sous-populations à fraie lithophile et d'autres à fraie phytopile (HOLCIK et HRUSKA, 1967).

\section{II - LA FRAIE DES PERCIDES}

\section{A - La perche}

\section{1) Le contrôle de la période de} fraie par les variations de la température

THORPE (1977a) a regroupé 42 références sur les températures et les dates de reproduction de la perche dans l'ensemble de son aire géographique. La fraie commence au début de mars au sud et à la fin juin au nord de la zone de répartition de la perche. Les températures qui accompagnent ce phénomène varient entre 5,6 et $14^{\circ} \mathrm{C}$. Les fraies les plus tardives correspondent aux températures les plus basses. Le seuil thermique nécessaire au déclenchement de la fraie diminue lorsque la latitude augmente : il se pourrait donc que ce seuil s'abaisse lorsque la fraie tarde à se produire (figure 4).
A la latitude de la France, la période de reproduction de la Perche peut varier en fonction de la taille du plan d'eau.

En 1983, les Perches ont frayé en avril dans les étangs du nord de la France, à $12^{\circ} \mathrm{C}$ environ. La même année, les perches des lacs subalpins se sont reproduites un mois plus tard, à des températures voisines de $10^{\circ} \mathrm{C}$. Ce retard peut s'expliquer par le fait que les lacs profonds se réchauffent plus lentement au printemps que les étangs ou les balastières car la thermocline ne s'établit qu'en juin (figure 5). Certains auteurs signalent que les classes d'âge peuvent se succéder sur les zones de frayères pendant plusieurs semaines: (HOLCIK, 1969 : THORPE, 1977b).

Dans le lac Léman, la fraie de la perche commence la dernière semaine d'avril lorsque le temps est beau, la température de l'eau atteint alors environ $12^{\circ} \mathrm{C}$ en surface.

Lorsque les conditions climatiques sont plus mauvaises, la fraie est retardée d'une quinzaine de jours et elle démarre à une température voisine de $10{ }^{\circ} \mathrm{C}$.

\section{2) L'emplacement et la composition des zones de frayères}

La perche dépose l'ensemble de ses oeufs en une seule fois. Ceux-ci sont reliés les uns aux autres par une gangue de mucus et ils forment un ruban dont la longueur varie entre 0,5 et 3,5 mètres suivant la taille de la femelle. Les rubans d'oeufs sont accrochés à divers substrats, plus particulièrement sur les branchages ou enroulés le long des tiges des plantes aquati- 

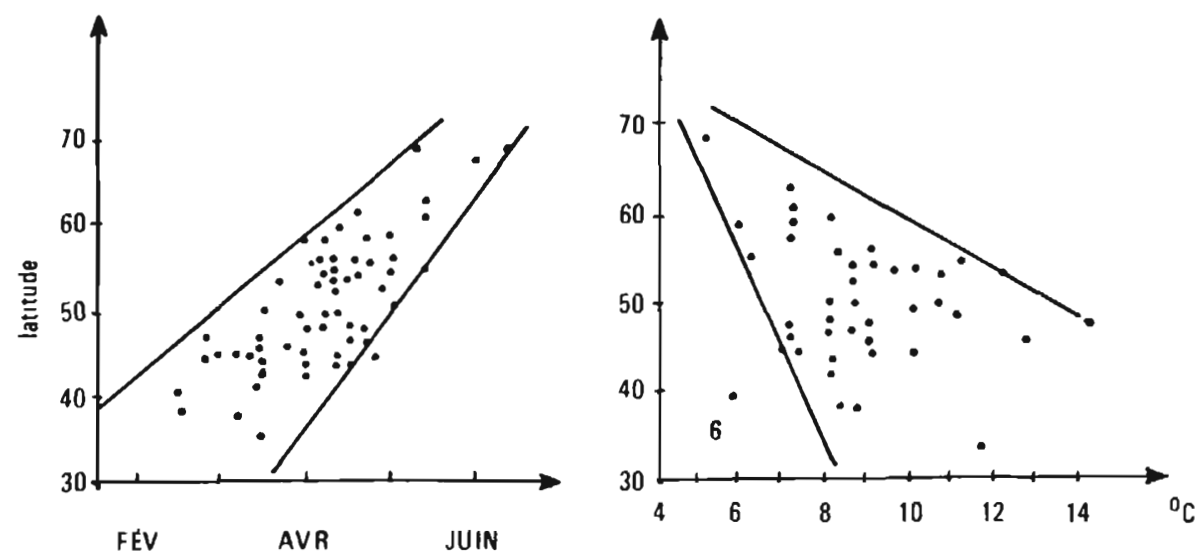

Figure 4 : Dates et températures de fraie chez la perche en fonction de la latitude (d'après THORPE, 1977a).

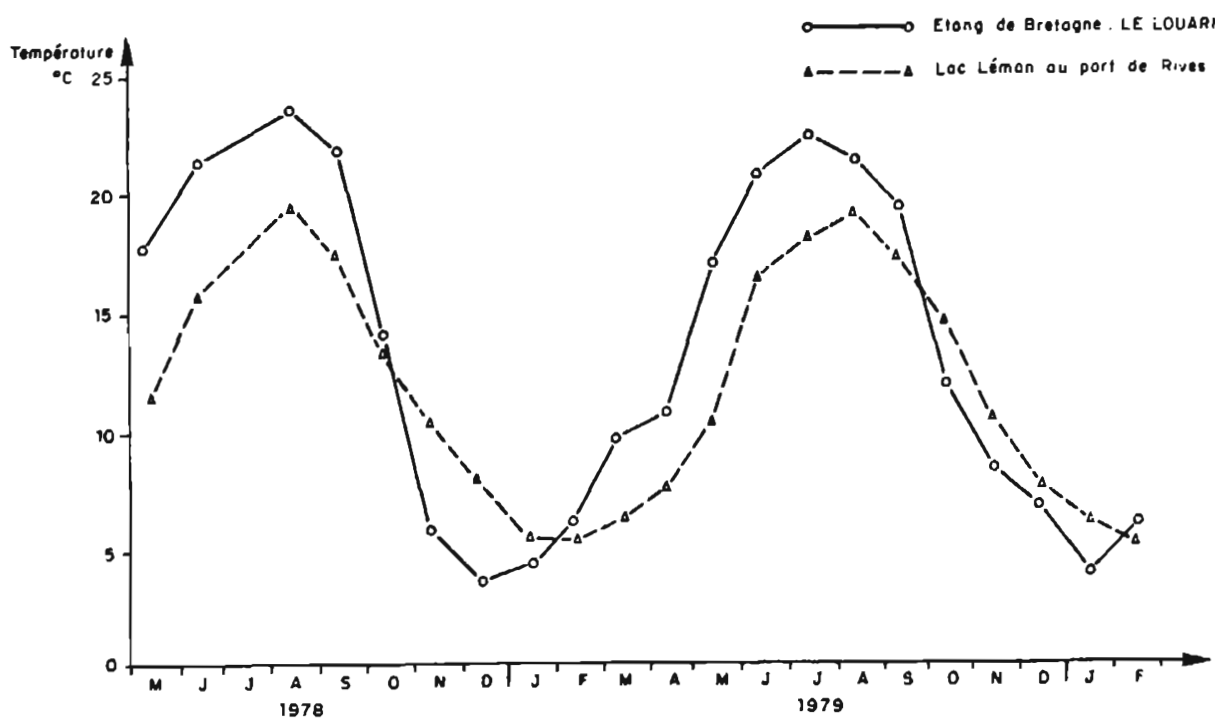

Figure 5 : Evolution annuelle de la température de surface d'un lac profond et de celle d'un étang.

ques. La profondeur à laquelle fraie la perche dépend de la taille du plan d'eau. Dans les étangs et les lacs de faible profondeur, les pontes se trouvent dans moins d'un mètre d'eau tandis que dans les grands lacs, les rubans sont déposés entre 3 et $10 \mathrm{~m}$ (tableau 3). La perche recherche les endroits abrités du vent pour frayer. Les populations peuvent changer de zones de frayères lorsque les vents dominants évoluent pendant la fraie 
Tableau 3 : Composition et profondeur des frayères naturelles chez la perche (perca fluviatilis).

\begin{tabular}{|c|c|c|c|c|}
\hline Auteur & Substrat de la frayere & $\begin{array}{l}\text { Profondeur } \\
\text { moyenne }\end{array}$ & Profondeurs extrenes & LdC \\
\hline LANG, 1981 & $\begin{array}{l}\text { Macrophytes, branchages } \\
\text { au fond calllouteux }\end{array}$ & 3,5 . & $1 d 10$ & Lénan \\
\hline GILLET, GERDFAUX, 19E? & Branchages & & 4 d $8=$ & Léuan \\
\hline $\begin{array}{l}\text { C.UMA'A, } 1977 \\
\text { UIROLYAINEN } 1940\end{array}$ & & & $\begin{aligned} 5 \text { d } 10 & =\end{aligned}$ & Hinderwere \\
\hline JONES, 1981 & Branchages & & Id $5=$ & Loch Leven \\
\hline GILLET, 19E3 & & & 1,5 d $2,5=$ & Paladru \\
\hline TREASURER, 1980 & Plantes diverses & $0,57(1976)$ & & Loch Xinord \\
\hline TREASURER, 1980 & Joncs & $\begin{array}{c}0,61 \\
0,79\end{array}$ & & Loch oayan \\
\hline GILLET, $198 \%-1983$ & Plantes diverses & & $0,1 \dot{d} 0,5=$ & Etang \\
\hline
\end{tabular}

(JONES, 1981). C'est vraisemblablement en raison des dégâts que provoqueraient les vagues déferlantes sur les rubans d'oeufs déposés près de la surface que les populations des grands lacs se sont adaptées à frayer profondément. La couche d'eau brassée par les vagues s'étend sur une hauteur qui est le double de celle des vagues. Les grandes tempêtes causent malgré tout des dégâts considérables sur les oeufs de perches (THORPE, 1977b).
3) L'influence des facteurs de l'environnement au cours de la période d'incubation.

Le pourcentage d'embryonnement des oeufs de la perche est généralement très élevé (tableau 4). La gangue du mucus qui entoure les oeufs serait une protection efficace contre la prédation par les invertébrés et les poissons. THORPE (1977b) signale que les cygnes sont partois prédateurs des oeufs de la perche.

La gamme des températures favorables au développement embryonnaire de la perche s'étend de 7 à

Tableau 4 : Pourcentage de fécondation des oeufs de perches sur les frayères.

\begin{tabular}{|c|c|c|}
\hline LdC & $\begin{array}{l}\text { Pourcentage de } \\
\text { fécondation }\end{array}$ & Auteur \\
\hline $\begin{array}{l}\text { Windernere, 1976-77 } \\
\text { Oneidd, 1976-17 } \\
\text { Cassidy, 1976-77 } \\
\text { Kinord, 1976 } \\
\text { Kinord, 1977 } \\
\text { Odruan, 1476 } \\
\text { Leiman, 1983 } \\
\text { Pdiadru, 1983 } \\
\text { Bdlastiere, 1982-83 }\end{array}$ & $\begin{array}{c}88-90: \\
, 95 \vdots \\
, 96 \vdots \\
99,4 \vdots \\
99,7 \vdots \\
99,3 \vdots \\
94,8 \vdots \\
94,3 \vdots \\
94,6 \vdots \text { et } 91,1:\end{array}$ & $\begin{array}{l}\text { GUHA'A, } 1917 \\
\text { CLADY, 1976 } \\
\text { SCHNEIUER, } 1972 \\
\text { IREASURSR, 1980 } \\
\text { IREASUPER, } 1980 \\
\text { IREASURER, } 1980 \\
\text { GILLEY, 14R3 } \\
\text { GILLEI, 1983 } \\
\text { GILLET, } 1983\end{array}$ \\
\hline
\end{tabular}


$16{ }^{\circ} \mathrm{C}$. HOKANSON et KLEINER ont démontré qu'une élévation progressive de 0,5 à $1{ }^{\circ} \mathrm{C}$ par jour constitue le meilleur régime thermique pour le développement embryonnaire de la perche (1975). Le succès de la reproduction naturelle de la perche dépend beaucoup des facteurs climatiques, principalement du vent et de la température pendant les premières semaines de la vie lanvaire (CLADY, 1976). Dans les conditions les plus défavorables le recrutement des juvéniles de perche peut-être pratiquement nul (CRAIG et KIPLING, 1983).

\section{B - Le sandre}

Le sandre fraie plus tard que la perche, à des températures de 12 à $15^{\circ} \mathrm{C}$, en avril, en mai et parfois en juin dans les lacs les plus froids (DEELDER et WILLEMSEN, 1964). $\mathrm{Ce}$ poisson a la particularité de construire un nid, généralement avec des débris végétaux et des racines. Le mâle aère les oeufs et les protège de la prédation pendant l'incubation. Cette adaptation permet au sandre de se reproduire dans des milieux où la turbidité est forte et l'oxygénation médiocre car le mâle nettoie les oeufs et leur assure un certain renouvellement de l'eau. Dans les régions les plus nordiques de son aire de répartition, certaines populations dispersent leurs oeufs sans en assurer la garde (BALON et al., 1977).

Le sandre fraie de préférence sur des fonds sableux ou caillouteux, à des profondeurs variant de 0,5 à 17 m. (Dans les eaux stagnantes. les nids sont généralement situés à des profondeurs supérieures à trois mètres. Le pourcentage de fertilisation des oeufs du sandre est généralement élevé et les pertes peu importantes pendant le développement embryonnaire.

Les températures que supporte l'embryon s'étendent entre 9 et $24^{\circ} \mathrm{C}$. A l'éclosion, un éclairement supérieur à 50 lux est léthal pour l'alevin jusqu'à ce que sa pigmentation cranienne apparaisse (DEELER et WILLEMSEM, 1964), ce qui pourrait expliquer pourquoi le sandre, qui prospère dans certains milieux eutrophes s'adaptent mal dans les lacs oligotrophes où l'eau est très transparente.

\section{III - LA FRAIE DU BROCHET}

\section{A - La date de reproduction et son contrôle par l'environnement}

La fraie du brochet commence à la fin de l'hiver, juste après le dégel dans les zones les plus nordiques. Les dates citées dans la littérature pour la fraie de ce poisson sont comprises entre la fin de février et le mois de juin du sud au nord de son aire de répartition (tableau 5 ).

En France, la plupart des populations vivant en étang frayent en mars, celles des lacs et des grands réservoirs se reproduisent un mois plus tard en raison du réchauffement plus tardif de ce type de milieu (figure 5).

La durée de la fraie, pour chaque population est comprise entre quelques jours et un mois. Dans un lieu 
donné, le déclenchement de la fraie ne varie pas de plus de 15 jours entre une année précoce et une année tardive (HUET, 1976).

Les températures de l'eau, relevées par différents auteurs pendant la reproduction du brochet s'étalent de 2 à $20^{\circ} \mathrm{C}$ (tableau 6). II semble cependant que l'oviposition se produise rarement au-dessous de $7^{\circ} \mathrm{C}$ ou audessus de $14^{\circ} \mathrm{C}$. A l'époque de la fraie du brochet, les fluctuations journalières de températures sont très importantes dans les zones peu profondes où cette espèce dépose ses oeufs. De plus, l'évolution thermique de ces couches d'eau est très rapide, au printemps, lorsque les conditions météorologiques varient, ce qui pourrait expliquer, en partie, l'importance de l'intervalle des températures citées dans la littérature.

Les crues semblent jouer un rôle de première importance dans la migration des géniteurs vers les zones de frayères et le déclenchement de la fraie. Dans beaucoup de régions, le brochet fraye après la fonte des neiges et profite des crues importantes et prolongées, associées au réchauffement printanier (MACHNIAK, 1975a).

\section{B - Structure et localisation des frayères}

Les frayères les plus couramment décrites pour le brochet sont des zones marécageuses, des fossés, des baies

Tableau 5 : Dates de reproduction du brochet (Esox lucius L.) d'après une synthèse bibliographique de K. MACHNIAK (1975a).

\begin{tabular}{|c|c|c|c|c|c|c|}
\hline Mors & liveler & Mase, & AUTII & M.11 & נル & H, III I \\
\hline 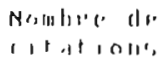 & " & 14 & 2x: & 21 & 6 & $1(n), 1,1$, \\
\hline
\end{tabular}

Tableau 6 : Températures de fraie $\left({ }^{\circ} \mathrm{C}\right)$ du brochet (Esox lucius L.) d'après une synthèse bibliographique de K. MACHNIAK (1975a).

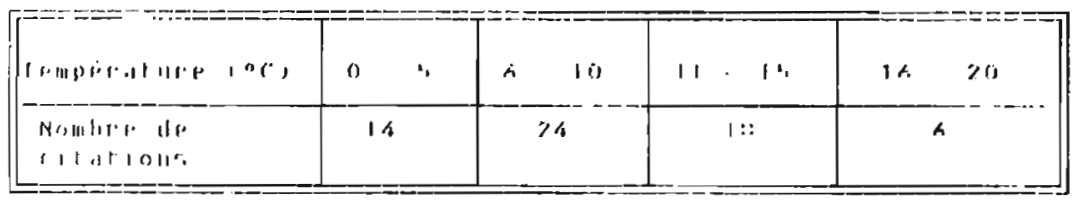

Tableau 7 : Profondeurs $(m)$ des frayères du brochet (Esox lucius L.) d'après une synthèse bibliographique de K. MACHNIAK (1975a).

\begin{tabular}{|c|c|c|c|c|c|}
\hline Profondeur on im & $0 \% .4$ & $\because, 5,0$ & $\therefore, 0, h$ & $101)$ & , 10\% \\
\hline Nonbre de $(1,11,13$. & $1 h$ & $1 / 6$ & 4 & 1 & 2 \\
\hline
\end{tabular}


peu profondes ou des zones d'inondation. Dans ces sites les oeufs de brochets sont déposés sous une faible épaisseur d'eau, entre 10 et 75 cm (voir tableau 7).

Les brochets ne recherchent pas de manière exclusive, certaines espèces de végétaux pour leur fraie. Leurs oeufs ont été observés sur toutes sortes de plantes: Elodée Myriophylle, Fontinale, Hippuris, Carex, Phagmite et diverses autres graminées (TONER et LAWLER, 1969). Suivant l'importance des crues, (CHAPLEAU et THELLEN, 1980) les brochets utilisent différentes ceintures de végétation, caractérisées par différentes associations végétales. Des oeufs de brochets ont aussi été découverts sur des substrats très divers, comme des aiguilles de Pin, des branches de Pin ou du foin coupé (synthèse de SOUCHON, 1983). Le brochet semble assez éclectique pour le choix de son substrat de fraie, mais il marque une préférence pour les structures denses et rares.

Lorsqu'une retenue vient d'être mise en eau, les brochets trouvent de vastes zones de frayères dans la végétation nouvellement immergée. En général, les premières années d'existance des réservoirs sont caractérisées par une très bonne réussite de la reproduction de brochet. Par la suite, le succès de la fraie du brochet décroit et peut même devenir nul losque les fluctuations de niveau font disparaître toute la végétation de la zone littorale et augmentent la turbidité de l'eau (NELSON, 1978 : GROEN et SCHROEDER, 1978). Les difficultés du brochet pour trouver dans les lacs et les réservoirs des zones de frayères naturelles sont d'autant plus importantes que la surface de la zone littorale est réduite par rapport à la surface totale en eau (MACHNIAK, 1975a). En effet le brochet disperse ses oeufs sur des surfaces plus importantes que la plupart des autres poissons lacustres. Les frayères naturelles du brochet occupent souvent des surfaces de plusieurs hectares (WILKONSKA et ZUROMSKA, 1967 : GRAVEL et DUBE, 1980). La densité des oeufs sur les frayères atteint au maximum quelques dizaines d'unités par m2 (TONER et LAWLER 1969 : FORTIN et al., 1982).

Une deuxième catégorie de frayères pour le brochet a été décrite dans les lacs de Mazurie. II s'agit de zones littorales et de hauts fonds recouverts de plantes aquatiques (Elodée, Potamogeton) situées à des profondeurs de 2 à $7 \mathrm{~m}$. Ces frayères seraient utilisées plus tardivement que les frayères du premier type, par des poissons de plus grande taille WILKONSKA et ZUROMSKA, 1967. KIPPLING et FROST rapportent le même type de phénomène pour le lac Windermere (cité par TONER et LAWLER, 1962).

\section{C - Comportement de fraie du brochet}

Les brochets sont capables d'accomplir des migrations de plusieurs $\mathrm{km}$ pour atteindre des zones de frayères. Ces migrations sont généralement nocturnes. Le réchauffement de l'eau et l'élévation du niveau se- 
raient les deux facteurs externes contrôlant le déroulement de ce phénomène (MACHNIAK, 1975a).

Les brochets frayent pendant la journée, ou seulement l'après-midi lorsque la température de l'eau est descendue très bas pendant la nuit. L'ensoleillement et l'absence de vent semblent stimuler le comportement de fraie des brochets. Chaque femelle dépose ses oeufs isolément, accompagnée par un ou quelques mâles. La femelle accomplit une série d'ovipositions, en déposant chaque fois quelques dizaines d'oeufs et en se déplaçant de quelques mètres entre chaque lâché d'oeufs (MACHNIAK, 1975a).

\section{D - Influence des facteurs ex- ternes au cours de l'incubation et la vie larvaire}

Le succès de la fraie du brochet dépend pour une très grande partie des conditions météorologiques. Le développement embryonnaire de cette espèce dure environ $120^{\circ} \mathrm{C} /$ jours, soit une à trois semaines suivant la température de l'eau. Pendant cet intervalle de temps, l'oeuf reste soumis à la prédation, aux variations de température et au risque d'exondation. Une décrue trop rapide provoque la mort des embryons qui sont déposés sous une faible hauteur d'eau. Les chutes de température peuvent provoquer des mortalités embryonnaires importantes (FORTIN et al., 1982) car la température léthale inférieure des embryons est de $3^{\circ} \mathrm{C}$ (HASSLER, 1982). Selon HOKANSON et al., 1973), les températures optimales pour l'incubation sont comprises entre 6 et $16^{\circ} \mathrm{C}$. Dans certains lacs, la prédation des oeufs peut prendre des proportions importantes. Les prédateurs sont des poissons (la perche. le vairon, etc...) des insectes et même des oiseaux. La turbidité de l'eau peut aussi constituer un facteur de mortalité important, lorsque la sédimentation atteint plusieurs millimètres pendant la durée de l'incubation (MACHNICK, 1975a).

Les pourcentages de survie des oeufs de brochets, cités dans la littérature sont très variables. $95 \%$ d'éclosion dans le lac Georges et $98 \%$ de mortalité dans le lac Straken (TONER et LAWLER, 1969). Dans les petits étangs aménagés pour l'élevage larvaire, la survie des larves est souvent supérieure à $30 \%$ (BRY et GILLET, 1980) mais dans les conditions naturelles, la survie des juvéniles est beaucoup plus faible (SOUCHON, 1983 pour synthèse). Le brochet est probablement l'espèce dont les frayères naturelles sont les plus menacées car ce poisson a besoin de surfaces importantes pour déposer ses oeufs. D'autre part, les meilleures frayères pour ce poisson sont les parties marécageuses de la zone littorale, qui deviennent de plus en plus rares.

\section{IV - LA FRAIE DES \\ SALMONIDES LACUSTRES}

Ces espèces se reproduisent en automne et en hiver. La fraie de ces poissons n'est pas contrôlée par une 
élévation de température comme chez les cyprinidés. La photopériode est le principal facteur externe qui contrôle le déroulement du cycle reproducteur des salmonidés.

\section{A - La truite de lac (Salmo trutta)}

Cette espèce se reproduit généralement dans les affluents des lacs, sur les mêmes zones de frayères que les populations sédentaires de truites de rivière. La fraie de la truite de lac ne peut se dérouler d'une manière satisfaisante que si l'estuaire et le cours inférieur des affluents ne sont pas barrés par des obstacles qui empêcheraient ce poisson d'accéder à ses zones de frayères. Dans le lac de Garde, en Italie, une espèce de truite, Salmo carpio, se reproduit dans le lac lui-même (BEHNKE, 1972).

Dans le Léman, des expériences de marquage, réalisées du côté français et du côté suisse montrent l'existence de phénomènes de homing pour la truite de lac.

\section{B - Les corégones}

Ces espèces présentent une grande diversité dans leur manière de frayer. Cette diversité concerne à la fois la période de reproduction qui varie entre le début de l'automne et la fin de l'hiver et les sites des frayères.

\section{1) La période de reproduction et la localisation des frayères}

En Scandinavie, il existe des espèces de corégones qui remontent les cours d'eau pour frayer sur des gra- vières,.des espèces qui frayent dans la zone littorale et des espèces qui frayent dans la zone pélagique des lacs. Les espèces qui se reproduisent dans les zones peu profondes, frayent généralement en automne et celles qui frayent en zone pélagique, l'hiver. La même espèce peut frayer différemment d'un lac à l'autre. En Suède le lavaret dépose ses oeufs en zone littorale ou pélagique et le Peled en rivière ou en zone pélagique (LINDSTROM, 1970).

Dans la région alpine, il existe des corégones qui frayent en zone pélagique comme la bondelle du lac de Neuchâtel qui se reproduit en janvier et en février au-dessus de fonds de 60 à $120 \mathrm{~m}$. Le blaufelchen du lac de Constance fraye dans les mêmes conditions tandis que la palée de Neuchâtel fraye en décembre sur la zone littorale dans une gamme de profondeur allant de $1 \mathrm{~m}$ à plus de $10 \mathrm{~m}$ (DOTTRENS et QUARTIER, 1941). Cette dernière espèce a été acclimatée dans plusieurs lacs fançais : le Léman, Saint Point, etc.

Le lavaret qui vit dans le lac du Bourget et dans celui d'Aiguebelette fraye sur les galets dans un mètre d'eau. Sa fraie est d'autant plus précoce que l'hiver est froid (LEROUX, 1928). II semble que les corégones n'aient pas des exigences très strictes vis-à-vis de la profondeur de leurs frayères. Chez le grand corégone d'Amérique, les profondeurs des frayères varient entre 1 et $30 \mathrm{~m}$ (tableau 8). D'autre part lorsqu'un corégone est introduit dans un nouvel habitat, il change facilement de zone de frayères : la palée de Neuchatel, in- 
troduite dans de nombreux lacs en France et en Suisse, fraye tantôt sur le bord, dans moins d'un mètre d'eau, tantôt sous 3 à 15 mètres d'eau (Léman, $S^{t}$ Point). C'est vraisemblablement l'existence d'un substrat favorable qui détermine le choix des zones de frayères chez les corégones. Certains auteurs supposent que les populations de corégones présentent une certaine fidélité à leurs zones de frayères, mais l'existence de homing n'a pas encore été mise en évidence (MACHNIAK, 1975b).

Les dates de fraie ainsi que les températures qui accompagnent ce phénomène varient beaucoup. Pour le grand corégone d'Amérique, MACHNIAK (1975b) a rassemblé plu- sieurs dizaines de références: les dates de reproduction sont comprises entre septembre et janvier et les températures accompagnant la fraie varient entre 0,5 et $10^{\circ} \mathrm{C}$ (tableaux 9 et 10). II semble que d'une manière générale, les populations nordiques frayent plus tôt que les populations vivant à nos latitudes. D'autre part, le refroidissement de l'eau stimulerait la migration des poissons vers les zones de frayères.

La fraie d'une espèce donnée dure environ un mois. Les mâles arrivent les premiers sur les zones de frayères et ils y demeurent pendant toute la période de reproduction tandis que les femelles ne viennent sur les frayères que pour l'oviposition. En rai-

Tableau 8 : Dates de reproduction du corégone (Coregonus clupeaformis) d'après une synthèse bibliographique de K. MACHNIAK (1975b).

\begin{tabular}{|c|c|c|c|c|c|}
\hline Mo1s & jeptrmbro & oci.oure & Nourmble & [ & ין יו וניוזוd א. \\
\hline $\begin{array}{l}\text { Nombre de } \\
\text { ritations }\end{array}$ & 4 & 13 & 2 & $1 !$ & 1 \\
\hline
\end{tabular}

Tableau 9 : Températures de fraie $\left({ }^{\circ} \mathrm{C}\right)$ du corégone (Coregonus culpeaformis) d'après une synthèse bibliographique de K. MACHNIAK (1975b).

\begin{tabular}{|l|c|c|c|c|c|}
\hline rempérdiure (oc) & $0-2$ & $2-4$ & 46 & $6: 1$ & $\therefore 10$ \\
\hline $\begin{array}{l}\text { Nombre lif } \\
\text { ritations }\end{array}$ & 1 & 4 & 6 & 4 & 1 \\
\hline
\end{tabular}

Tableau 10 : Profondeurs des frayères $(\mathrm{m})$ du corégone (Coregonus clupeaformis) d'après une synthèse bibliographique de K. MACHNIAK (1975b).

\begin{tabular}{|c|c|c|c|c|c|c|c|c|}
\hline Profondeur en m & 0 & . 1 & 1 & ? & 5 & 10 & 10 & .40 \\
\hline Nombre te rellatione, & & 4 & & $1:$ : & & 12 & & h \\
\hline
\end{tabular}


son de ce phénomène, le sex-ratio des individus capturés sur les zones de frayères est fortement déséquilibré en faveur des mâles (SLACK, 1969 : CHAMPIGNEULLE et al., 1983).

\section{2) L'action des facteurs externes au cours de l'incubation}

Les corégones dispersent leurs oeufs au-dessus du fond. La fraie peut se dérouler sur des substrats herbacés, sableux, caillouteux ou boueux. Selon LINDSTROM (1970) la nature du fond ne constituerait pas un stimulus important pour la fraie des corégones, mais la survie des oeufs est faible lorsqu'ils sont déposés sur des fonds boueux (ZAWISZA et BACKIEL, 1970).

La durée de l'incubation varie avec la température de l'eau. Le nombre de degrés-jour nécessaire à l'accomplissement de cette phase varie de 100 à 300 lorsque la température d'incubation s'élève de 1 à $10^{\circ} \mathrm{C}$ chez le lavaret (SALOJARVI, 1982). Les températures les plus favorables pour les embryons de corégones sont comprises entre 3 et $6{ }^{\circ} \mathrm{C}$. Au-dessus de $8^{\circ} \mathrm{C}$, les mortalités embryonnaires deviennent très importantes chez le whitefish (PRICE, 1935). Lorsque la température s'abaisse au-dessous de $2{ }^{\circ} \mathrm{C}$, la survie des oeufs de marène diminue, d'après les observations réalisées sur les frayères naturelles des lacs de Mazurie (ZUROMSKA, 1982).

La teneur en oxygène dissous peut constituer un facteur limitant pour le développement des oeufs de corégones, FLUCHTER (1980) signale que l'embryon manque d'oxygène dès que la concentration de ce gaz de- vient inférieure à $8 \mathrm{mg} / \mathrm{l}$. La survie des oeufs de corégones est pratiquement nulle sur les fonds vaseux des lacs eutrophes en raison du déficit en oxygène qui règne au niveau des sédiments, en Finlande (LAHTI et al., 1979). L'eutrophisation, ainsi que la prédation et les mauvaises conditions climatiques sont responsables de pertes supérieures à $95 \%$ pendant le développement embryonnaire chez la marène et le lavaret des lacs de Mazurie. Dans les lacs les plus eutrophes, aucun oeuf ne parvient à éclore et les stocks de corégones ne sont plus entretenus que par le déversement de laves produites en écloserie (WILKONSKA et ZUROMS$K A, 1982)$.

Les oeufs de corégones sur les frayères sont la proie des invertébrés comme les aselles et les trichoptères ainsi que des poissons. NUMAN (1970) signale qu'une lotte capturée dans le lac de Constance avait l'estomac rempli par 1800 oeufs de corégones. Dans le lac Léman nous observons chaque année un grand nombre d'oeufs de corégones dans l'estomac des lottes capturées sur les zones de frayères pendant tout le mois de janvier.

La turbidité de l'eau et la sédimentation qui en résulte pourrait constituer un facteur de mortalité important dans les lacs eutrophes et les réservoirs. FUDGE et BODALY (1984) ont trouvé une forte corrélation entre le pourcentage de mortalité des oeufs et l'intensité de la sédimentation daris un réservoir où l'élévation du niveau d'eau avait entrainé une forte érosion des berges. Nous avons observé 
dans l'espace de 4 semaines, un dépôt de sédiment très important sur les oeufs dans deux lacs eutrophes où les populations de corégones ont fortement décliné. Nous ne constations aucun dépôt, pendant la même période sur des oeufs placés dans un lac oligotrophe où la population de corégones prospère.

Lorsque les lacs s'eutrophisent, la qualité des oeufs de corégones à tendance à diminuer (WILKONSKA et ZUROMSKA, 1982). Cette décroissance de la fécondabilité des oeufs s'accompagne d'une diminution de la teneur en lipides, en protéines et en calories du vitellus de l'oeuf (KAMLER et al., 1982). Ces auteurs supposent que la désoxygénation des couches profondes des lacs eutrophes obligent les géniteurs de corégones à passer l'été dans les eaux superficielles où la température est trop élevée pour permettre un déroulement satisfaisant de la gamétogénèse.

\section{C - L'omble chevalier}

Cette espèce dont l'aire de répartition s'étend sur l'ensemble des régions arctiques, se compose d'un grand nombre de races notamment dans les lacs subalpins où des populations d'ombles chevaliers ont été isolées depuis la fin des glaciations quaternaires.

Les habitudes de fraie de l'omble chevalier diffèrent d'une population à l'autre. Certains lacs, comme le Windermere, possèdent même plusieurs populations d'omble chevalier, qui vivent en isolement reproducteur. II existe dans ce lac un stock d'ombles qui se reproduit en automne sur la zone littorale, à des profondeurs comprises en 1 et $3 \mathrm{~m}$. Un deuxième stock se reproduit au printemps, en eau profonde, entre 15 et $20 \mathrm{~m}$. Une partie de la population qui se reproduit en novembre, remonte la rivière Beathay pour aller frayer dans une zone de gravière, le Purdom'Dub (FROST, 1965).

En général les populations d'ombles qui frayent à la fin de l'hiver et au début du printemps se reproduisent à des profondeurs importantes. Les basses températures qui se maintiennent toute l'année à de telles profondeurs sont favorables pour l'incubation des oeufs de l'omble chevalier.

Cette phase dure plusieurs mois et elle doit se dérouler aux alentours de $5^{\circ} \mathrm{C}$. En raison de ces exigences thermiques, il n'existe pas de population frayant en rivière dans la partie la plus méridionale de l'aire de répartition de ce poisson, c'est-à-dire dans les Alpes. En effet dans ces régions, la température de l'eau des rivières risquerait d'atteindre un niveau trop élevé pour permettre aux alevins d'y subsister jusqu'à l'âge de la dévalaison.

La fraie d'une population donnée dure environ un mois, (FROST, 1965), mais il existe toute une gamme de populations qui se reproduisent depuis le mois de septembre jusqu'au mois d'avril. Dans certains lacs de Bavière la fraie a lieu au cours de l'été (JOHNSON, 1980).

La profondeur choisie par l'omble chevalier pour déposer ses oeufs va- 
rie entre 1 et $100 \mathrm{~m}$ suivant les populations et les lacs. Le substrat des zones de frayères présente moins de variabilité que la profondeur ou la date choisie pour la fraie.

L'omble construit un nid de quelques dizaines de centimètres sur des fonds de galets et d'éboulis. La granulométrie des blocs associés aux oeufs de l'omble varie généralement entre 3 et $20 \mathrm{~cm}$ (FROST, 1965). II existe cependant certains lacs où des oeufs d'ombles chevaliers ont été trouvés sur des fonds boveux ou sableux. L'omble recouvre ses oeuis après la fraie, la profondeur moyenne d'enfouissement est d'environ $10 \mathrm{~cm}$ (JOHNSON, 1980). Les observations réalisées en soucoupe pendant la fraie des ombles au Léman laissent supposer que ceux-ci déposent simplement leurs oeufs sur les galets.

L'omble chevalier manifeste une certaine fidélité à ses zones de frayère. Dans deux lacs suédois, le Storjoute et le Torrön, le niveau de l'eau fut réhaussé de $10 \mathrm{~m}$ à la suite de l'installation de barrages hydroélectriques. Les populations d'ombles chevaliers continuèrent en majorité à utiliser leurs anciennes zones de frayères littorales, bien que la profondeur moyenne de ces zones soit passée de $1 \mathrm{~m}$ à plus de $10 \mathrm{~m}$ (JOHNSON, 1980). Sur le lac Windermere, FROST (1963) a réalisé une expérience de marquage à partir de géniteurs capturés sur les zones de frayères. Les recaptures ont permis de conclure que les animaux revenaient se reproduire sur les mêmes zones de frayères d'année en année. Les ombles qui se reproduisent en automne sur le littoral sont toujours recapturés à cette date, sur ce type de frayères et il en est de même pour la population qui se reproduit au printemps, en eau profonde. Dans le cas de la population qui fraye en automne, deux zones de frayères éloignées de quelques $\mathrm{km}$ ont été étudiées. Les recaptures d'ombles marqués ont toujours été réalisées sur la frayère où les poissons avaient été capturés initialement. Des alevins ont aussi été déversés dans un affluent du lac Windermere où les ombles n'étaient jamais venus se reproduire auparavant. Deux ans plus. tard, des géniteurs étaient capturés dans le ruisseau. Ces observations permettent de supposer que l'omble chevalier a la capacité de mémoriser la zone de frayère où il est né.

Le développement embryonnaire de l'omble chevalier requiert 400 degrés-jours, ce qui correspond à une durée d'incubation de plus de trois mois lorsque la température de l'eau se situe aux alentours de $4^{\circ} \mathrm{C}: \mathrm{La}$ température léthale supérieure des embryons est de $12{ }^{\circ} \mathrm{C}$, mais le taux de mortalité augmente fortement dès que l'eau d'incubation dépasse $8^{\circ} \mathrm{C}$ (SWIFT, 1965).

Pendant l'incubation, les oeufs de l'omble sont consommés par plusieurs espèces de poissons: la truite, l'anguille et l'omble chevalier luimême. Lorsque les oeufs sont déposés sur la zone littorale ils sont aussi la proie de plusieurs espèces d'oiseaux aquatiques (FROST, 1952). De nombreuses lottes ont été observées en plongée sur les frayères des om- 
bles à Annecy et au Léman. Certains de ces poissons avaient des oeufs d'ombles dans leurs estomacs.

Dans les lacs eutrophes, les oeufs de l'omble chevalier se développent difficilement dans les zones profondes où l'oxygène se raréfie. Ce phénomène est encore accentué par le colmatage des frayères dû à l'envasement des lacs eutrophes. Certains micropolluants ont une action défavorable sur la reproduction des ombles, c'est le cas des polychlorobiphényles. Ces substances s'accumulent dans les oeufs et sont responsables de mortalités embryonnaires importantes (MONOD, 1983).

Dans certains lacs, un programme de restauration des frayères de l'omble chevalier a été mis en oeuvre. Dans le lac d'Annecy plusieurs milliers de tonnes d'éboulis ont été déversés sur d'anciennes frayères envasées. Par la suite des géniteurs d'ombles chevaliers ont été détectés sur ces sites.

\section{V - LA FRAIE DE LA LOTTE (LOTA LOTA)}

Ce gadidé se reproduit en hiver. Dans les lacs subalpins français la lotte commence à frayer à la fin de février (vers le 15.02 à Annecy et au Bourget et vers le 1.03 au Léman). La fraie de chaque population s'étale sur un mois environ.

Les frayères de la lotte sont situées à des profondeurs importantes. $30 \mathrm{~m}$ à Annecy et $60 \mathrm{~m}$ au Bourget (LE-
ROUX, 1928) et plus de $100 \mathrm{~m}$ au Léman. Dans les rivières, les lottes présentent un comportement différent : elles viennent près du bord en hiver et elles se reproduisent vraisemblablement à faible profondeur.

\section{VI - SYNTHESE DES DONNEES SUR LA FRAIE DES POISSONS LACUSTRES}

Les données présentées dans cet article permettent de dresser un tableau synthétique des caractéristiques des frayères des principales espèces de poissons lacustres, de leurs périodes de fraie et de la dose thermique, en degrés-jours,qui détermine la durée d'incubation (tableau 11).

La majorité des poissons lacustres fraye dans la zone littorale, à l'exception de la truite, de la lotte et de certaines souches d'ombles chevaliers et de corégones.

L'incubation des oeufs requièrt quelques jours ou plusieurs mois suivant les espèces. L'allongement de la durée du développement embryonnaire accroit la vulnérabilité des espèces aux phénomènes de marnage, au colmatage des frayères et à la prédation de leurs oeufs. Par ailleurs, les poissons, tel que le brochet, qui dispersent leurs oeufs sur de grandes surfaces et qui sont relativement exigeants pour le choix du substrat de fraie sont plus menacés par la dégradation de la zone littorale que des espèces très éclectiques comme le gardon ou la perche. 
Tableau 11 : Habitudes de fraie des principales espèces de poissons lacustres

\begin{tabular}{|c|c|c|c|c|c|}
\hline Fr,peres & $\begin{array}{c}\text { irajer: } \\
\text { Protrondeur et substrat }\end{array}$ & $\begin{array}{l}\text { udte de 'a trale et } \\
\text { Juree de l'incuodtion }\end{array}$ & $\begin{array}{l}\text { Uariations } \\
\text { entre souche }\end{array}$ & $\begin{array}{l}\text { Uariations entre } \\
\text { classe d'age }\end{array}$ & $\begin{array}{l}\text { Menorisation } \\
\text { des alres de } \\
\text { trayeres }\end{array}$ \\
\hline CArdon & $\begin{array}{l}0.1+1.5 \text { o sur herbiers } \\
\text { ou rerhers. }\end{array}$ & $\begin{array}{l}\text { début a a début juln } \\
100^{\circ} \mathrm{C} \mathrm{J} \text { a } 20^{\circ} \mathrm{C}\end{array}$ & - & 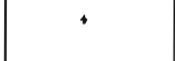 & . \\
\hline $\begin{array}{l}\text { Cyprinidpe, i entes } \\
\text { frdetronnees }\end{array}$ & 0,1 d s o sur herbiers & $\left\{\begin{array}{l}\text { dibut al d début roût } \\
100^{\circ} \mathrm{C} \mathrm{J} \text { d } 20^{\circ} \mathrm{C}\end{array}\right.$ & $\cdots$ & ? & 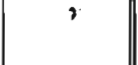 \\
\hline Ferche & $\begin{array}{l}0,2 \text { d } 10 \text { sur un } \\
\text { substrd: bu:ssonnant }\end{array}$ & détut aur j fin & - & . & - \\
\hline sonore & id 7 dans un nid & début auril d tin וd & - & - & - \\
\hline Brochet & $\begin{array}{l}0,2 \text { d } 1 \text { a sur los } \\
\text { nerbiters }\end{array}$ & $\left\{\begin{array}{l}\text { : } 20^{\circ} \mathrm{C} \text {. J a a } 10^{\circ} \mathrm{C} \\
\text { fin fiurier a i }\end{array}\right.$ & - & 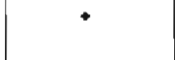 & , \\
\hline Porégones & $\begin{array}{l}1 \text { d plusieurs centalines } \\
\text { te a sir diver's substrats }\end{array}$ & $\left\{\begin{array}{l}\text { futoune et hiver } \\
300^{\circ} \mathrm{C} \mathrm{J} \text { d } 50 \mathrm{C}\end{array}\right.$ & - & $\boldsymbol{7}$ & 1 \\
\hline onble theudlier & $\begin{array}{l}\text { I a } 100 \text { sur dps galpte, } \\
\text { et éboulis }\end{array}$ & $\begin{array}{l}\text { Aistonne, hiupr et prin- } \\
\text { telos } 400^{\circ} \mathrm{C} . J \text { a } 6^{\circ} \mathrm{C}\end{array}$ & + & - & $\leftarrow$ \\
\hline Iruste de lac & $\begin{array}{l}\text { en riviere, sur des } \\
\text { gravieres }\end{array}$ & $\left\{\begin{array}{l}\text { tin occodre d debut } \\
\text { féurier } 400^{\circ} \mathrm{C} . \mathrm{J} \text { d } 4^{\circ} \mathrm{C}\end{array}\right.$ & - & - & - \\
\hline
\end{tabular}

Ces deux derniers poissons acceptent toutes sortes de substrats pour frayer et de plus ils concentrent leurs oeufs sur de petites surfaces.

L'omble chevalier, le corégone, le gardon, l'ablette et probablement d'autres espèces comprennent plusieurs souches qui diffèrent entre elles par le lieu ou la période de fraie.

Parfois plusieurs de ces souches arrivent à cohabiter dans le même lac, c'est le cas pour l'omble chevalier dans le lac Windermere et dans beaucoup de lacs scandinaves $(\mathrm{JOHN}$ SON, 1980). Selon HOLCIK et HRUSKA (1966), cette diversité serait le propre des espèces d'origine récente, qui possèdent une grande plasticité et qui peuvent s'adapter facilement à differents environnements.

Cette variabilité devrait être prise en compte par les gestionnaires. Par$\mathrm{mi}$ les souches nordiques d'ombles chevaliers et de corégones, celles qui se reproduisent en rivière ne de- vraient pas être choisies pour les repeuplements des plans d'eau français où ce mode de reproduction n'existe pas. Dans les lacs en voie d'eutrophisation, les souches qui frayent dans la zone littorale paraissent plus appropriées que celles qui se reproduisent dans l'hypolimnion où l'oxygène pourrait devenir déficitaire. A l'opposé, dans les retenues à niveau variable, ce sont les souches qui frayent dans les zones profondes dont les oeufs pourraient échapper à l'exondation.

Le phénomène de "homing" a été mis en évidence chez certaines populations de gardons et d'ombles chevaliers. II est suspecté pour certaines populations de corégones et de brochets (MACHNIAK, 1975 a et b). II se pourrait que le phénomène de mémorisation des frayères soit assez fréquent pour les poissons lacustres, ce qui constituerait une donnée importante pour la gestion des populations de poissons. 


\section{REFERENCES BIBLIOGRAPHIQUES}

Balon E.K., 1975. Reproductive guilds of fishes: a proposal and definition. J. Fish. Res. Board Can., 32, 821-864.

Balon E.K., Momot W.T. and Regier H.A., 1977. Reproductive guilds of percids: results of the paleogeographical history and ecological succession. J. Fish. Res. Board Can. 34 (10), 1910-191.

Behnke R.J., 1972. The systematics of samonid fishes of recently glaciated lakes. J. Fish. Res. Board Can., 9, 639671.

Bray E.S., 1971. Observations on -the reproductive cycle of the Roach (Rutilus rutilus) with particular reference to the effects of heated effluents. proc. 5th British Coarse Fish Conf., 93-99.

Bry C. et Gillet C., 1980. Reproduction du cannibalisme précoce chez le brochet (Esox lucius L.) par isolement des fratries Bull. Fr. Piscic., 277, 142-153.

Breton B., Horosewicz L., Bienarz K. and Epler p., 1980. Temperature and reproduction in tench: effect of a rise in the annual temperature regime on gonadotropin level, gametogenesis and spawning. II The female. Reprod. Nutr. Dev., 20 (4A), 1024.

Chapleau D., Thellen G., 1980. Aménagement de frayères de grands brochets, Esox lucius L., au Québec. Rapp. Rech. Faun. Minist. Loisir, Chasse, Pêche, Dir. Rech. faun. (Que), 52, 77-88.

Clady M.D., 1976. Influence of temperature and wind on the survival of early stages of yellow Perch, Percca flavescens. J. Fish. Res. Board Can., 33, 1887-1893.

Champigneulle A., Gerdeaux D. et Gillet C., 1983. Les pêches de géniteurs de corégone dans le Léman français en 1982. Bull. Fr. Piscic,, 90, 149-157.

Craig J.F. and Kipling C., 1983. Reproduction effort versus the environment case histories of Windermere perch. Perca fluviatilis L. and Pike, Esox lucius L. J. Fish. Biol., 2 (5), 713-728.

Dauba F., 1981. Etude comparative de la faune des poissons dans les écosystèmes de deux réservoirs : Luzech (lot) et Chastang (Dordogne). Thèse de docteur ingénieur de l'Institut National polytechnique de Toulouse.

Deeler C.L. and Willemsen J., 1964. Synopsis o.f biological date on pikeperch. F.A.O. Fish. Synop., 28.

Dottrens E. et Quartier A., 1949. Les corégones du lac de Neuchâtel : étude biométrique. Rev. Suisse Zool., 56 (37), 689-731.

Dube J. et Gravel Y., 1978. Plan pilote d'aménagement intégré des ressources biologiques du territoire de la frayère du ruisseau Saint-Jean, Comté du Châteaugay, Québec. Ministère du Tourisme de la Chasse et de la Pêche, Montréal.

Easton K.W., Dalben I.P., 1980. The induced spawning and subsequent survival and growth of Roach, Rutilus rutilus L., Fish. Mgmt, 2 (2), 59-66.

Efimorea T.A., 1977. Influence of heated water discharges from Konakoreo Electric Power Station on sexual cycles of fish in Ivankov reservoir. Tr. Vses. Gidrobiol. O. Va., 21, 63-82. (en russe, cité par Horoszewicz 1981).

Fluchter J., 1980. Review of the present knowledge of rearing white-fish (Coregonidae) larvae. Aquaculture. 19, 191208.

Fudge R., Bodaly R., 1984. Postimpondment winter sedimentation and survival of lake whitefish (Coregonus clupeaformis) eggs in Southern Indian lake. Manitoba. Can. J. Fish. Aqua. Sci. 41 (4), 701-705. 
Fortin R., Dumont P., Fournier H. et al., 19822. Reproduction et force des classes d'âge du grand Brochet (Esox lucius L.) dans le Haut Richelieu et la baie Missisquoi. Can. J. Zool., 60, 227240.

Franklin D.R. and Smith L.L. Jr., 1960. Note on development of scale patterns in the northern pike (Esox lucius L.). Trans. Am. Fish. Soc., 89 (1), 83.

Frost W.E., 1952. Predators on the eggs of char in Windermere. Salmon Trout Mag., 143, 193-197.

Frost W.E., 1963. The homing of char, Salvelinus willughbii (Gunther) in Windermere. Anim. Behav. 11 (1), 75-82.

Frost W.E., 1965. Breeding habits of Windermere char, Salvelinus willughbii (Gunther) and their bearing on speciation in these fish. Proc. R. Soc. Edinb., B, 163, 232-284.

Gillet C., Billard R.. 1977 : Stimulation of gonadotropin secretion in goldfish by elevation or rearing temperature. Ann Biol. anim. Biochim. Biophys., 17, 673678.

Gillet C.. Roubaud P., 1983. Influence sur la survie jusqu'à éclosion des embryons de la carpe commune après traitement pendant la fécondation et le développement embryonnaire précoce par le carbendazine. Water Research $17(10,1343-1348$.

Gillet C., Roubaud P., 1986. Survie embryonnaire précoce de 9 espèces de poissons d'eau douce soumis à un choc de $\mathrm{pH}$ pendant la fécondation ou au cours des premiers stades du développement embryonnaire. Repro. Nutr. Develop. 26 (6), 1319-1333.

Goldspink C.R., 1977. The return of marked roach (Rutilus rutilus L.) to spawning grounds in Tjeukemeer, The Netherlands. J. Fish. Biol., 11 (5), 599604.

Gulidov M.V. and Popova K.S., 1981. The hatching dynamics and morphological features of larvae of Roach, Rutilus rutilus $L$. in relation to incubation temperature. J. Ichtyol., 1, 87-92.

Guma'a S.A., 1977. A study of young stages of perch (Perca fluviatilis L.) in Windermere. Ph. D. Thesis, University of Lancaster (cité par Treasurer).

Gravel Y., Due J., 1980. Plan de conservation du grand Brochet (Esox lucius L.) au lac Saint-Louis, Québec. Ministère du Tourisme, de la Chasse et de la Pêche du Québec, Montréal.

Groen, Schroeder, 1978. Effects of water level management on walleye and other coolwater fishes in Kansas reservoirs. Ann. Fish. Soc. Spec. Publ. 11. 278-283.

Handerson B., 1985. Factors affecting growth and recrutment of yellow perch, Perca flavescens Mitchill, in South Bay, Lake Huron. J. Fish. Biol. 6 (4), 449458.

Hassler T.J., 1982. Effect of temperature on survival of northern pike embryos and yolk sac larvae. Progr. Fish-Cult., 44 (4), 174-178.

Hokanson K.E., McCormick J.H., Jones B.R., 1973. Temperature requirements for embryos and larvae of the northern pike, Esox lucius L., Trans. Am. Fish. Soc., 102 (1), 89-100.

Hokanson K.E. and Kleiner C.F., 1975. Effects of constant and rising temperature on survival and development rates of embryonic and larval yellow perch, perca flavescens (Mitchill). In : The early life history of fish, J.H.S. Blaxter (Ed.), Springer Verlag, Berlin, 437-448.

Holcik J. and Hruska V., 1966. On the spawning substrate of the roach (Rutilus rutilus L.) and Bream (Abramis brama L.) and notes on the ecological Characteristic of some european fishes. Vest. Cesk. Spol. Zool., 30 (1), 22-29.

Holcik J., 1969. The natural history of perch, Perca fluviatilis L. in the Klicava 
reservoir. Pr. Lab. Ryb. Hydrobiol., 2. 269-305.

Horoszewicz L., Bieniarz K., Epler p., 1981. Effect of different thermal regimes on reproductive cycles of tench, Tinca tinca L. : Part IV. Duration and temperature of spawnings. Polsk. Arch. Hydrobiol., 28 (2), 207-16.

Huet M., 1976. Reproduction, incubation et alevinage du brochet (Esox lucius L.). E.I.F.A.C., Tech. Pap. 25, 147-163.

Jaoul A., Roubaud P., 1982. Résistance de l'oeuf de carpe commune (Cyprinus carpio L. Cyprinidae) à des chocs thermiques chauds ou froids. Can. J. Zool. 60 (12), 3409-3419.

Jones D.H., 1982. The spawning of perch, Perca fluviatilis $L$. in loch Leven, Kinross, Scotland. Fish. Manage., 13 (4), 139-151.

Johnson L., 1980. The arctic charr, Salvelinus alpinus $L$., In : Charrs, salmonid fishes of the genus Salvelinus: E.K. Balon (Ed.) : W. Junk, The Hague, 18-92.

Kamler E., Zuromska H., Nissinen T., 1982. Bioenergetical evaluation of environmental and physiological factors determining egg quality and growth in Coregonus albula L.. Pol. Arch. Hydrobiol., 29, 123-158.

Kennedy M. and Fitzmaurice P., 1968. The biology of the Bream, Abramis brama L., in irish waters. Proc. r. ir. Acad., 67 B, 95-156.

Kennedy M. and Fitzmaurice P., 1974. Biology of the Rudd, Scardinius erythropthalmus L., in irish waters. Proc. r. ir. Acad. 74 B, 245-308.

L'Abbee-Lund J.H., Vollestad L.A., 1985. Homing Precision of roach, Rutilus rutilus, in lake Arunngen, Norway. Environmental biology of fishes Vol. 13, $n^{0} 3,235-239$.

Lahti E., Oksman H. and Shemeikka P., 1979. On the survival of vendace eggs in different lake types. Aqua Fennica, 9. 62-67.

Lang C., 1981. Densité, localisation, taille et développement des chaines d'oeufs de la perche (Perca fluviatilis L.) dans le Léman de 1979-1981. Bull. Soc. Vaud. Sci. Nat., 360 (75), 257-265.

Lang C., 1987. Mortality of perch, Perca fluviatilis, estimated from the size and abundance of egg strands. J. Fish. Biol. 31, 717-720.

Le Louarn H., 1980. La polyculture d'étang en exploitation familiale, In : La pisciculture en étang : R. Billard (Ed.) : I.N.R.A., Paris, 293-286.

Leroux M., 1928. Recherches biologiques dans les grands lacs de Savoie. Lacs du Bourget et d'Annecy. Abry et $\mathrm{C}^{\text {ie }}$ éditeurs, Annecy.

Lindstrom T., 1970. Habitats of whitefish in some north swedish lakes at different stages of life history. In : Biology of Coregonid fishes, C.C. Lindsey and C.S. Woods (Eds). University of Manitoba Press, 461-480.

Machniak K., 1975a. The effects of hydroelectric development of the biology of northern fishes (reproduction and population dynamics). II Northern pike Esox lucius L., a literature review and bibliography. Fisheries and marine service, Technical report $n^{\circ} 528$, Canada.

Machniak K., 1975b. The effects of hydroelectric development of the biology of northern fishes (reproduction and population dynamics). I Lake whitefish, Coregonus clupeaformis. Fisheries and marine service. Technical report $n^{\circ}$ 527, Canada.

Martin D., Mengel L., Novotny J. and Walling C., 1981. Spring and summer water leveis in a Missouri River reservoir : effects of age 0 . fish and zooplankton. Trans. of Am. Fish. Soc. 110, 3703891. 
Mills C.A., 1980. Spawning and rearing eggs of the Dace, Leuciscus leuciscus, L.. Fish. Manage., 11 (2), 67-72.

Mills C.A., 1981. The spawning of roach, Rutilus rutilus $L$., in a chalk stream. Fish. Manage., 12(2), 49-54.

Monod G., 1983. Etude de la contamination chimique du lac Léman par les résidus organochlorés polychlorobyphéniles et D.D.T. Essai d'évaluation des risques pour la reproduction de l'omble chevalier. Thèse $3^{\mathrm{e}}$ cycle, Univ. Cl. Bernard, Lyon I, 164 p.

Nelson W., 1978. Implications of water management in lake Oahe for the spawing success of coolwater fishes. Am. Fish. Soc. Spec. Publ. 11, 154-158.

Numan W., 1970. The Blaufelchen of lake Constance, Coregonus wartmani under negative and positive influence of man. In : Biology of Coregonid fishes : C.C. Lindsey and C.S. Wood (Eds) : University of Manitoba Press, 531-552.

Peczalska A., 1968. Development and reproduction of roach (Rutilus rutilus. L.) in the Szczecin Firth. Polsk. Arch. Hydrobiol. 15 (2), 103-120.

Ponton D., Gerdeaux D., 1987. La population de gardons (Rutilus rutilus L.) du lac Léman en 1983-85. Structure en âge déterminisme du recrutement, analyse de la croissance. Bull. Fr. Pêche Piscic. 305, 43-53.

Price J.W., 1935. The embryology of the whitefish Coregonus clupeaformis (Mitchill). Ohio. J. Sci., 287-412.

Reznitchenko P., 1971. Problèmes des conditions thermiques optima de développement et de viabilité des oeufs de tanche, de brochet et d'esturgeon. Comptes-rendus de la Société des $\mathrm{Na}$ turalistes de Moscou. Zoologie Botanique. $\quad 2^{\mathrm{e}}$ semestre 1968-1969. Université de Moscou, 53-56.

Reznitchenko P., 1976. Règles générales de l'influence du facteur thermique sur l'embryogénèse des poissons.
Compte-rendu de la $2^{\mathrm{e}}$ conférence de Kiev. $1^{\text {re }}$ partie. Physiologie et Ecologie des Poissons. Acad. Sci. Ukr., Nankoua. D.

Saglio P., 1979. Communication chimique et migration reproductrice chez les salmonidés. Bull. Fr. pisci., 275, 7282.

Salojarvi K., 1982. Spawning ecology, larval food supplies and causes of larval mortality in the whitefish (Coregonus lavaretus L.). Polsk. Arch. Hydrobiol., 29, 159-178.

Slack H.D., 1969. The reproduction and fecundity of the Powan, Coregonus clupeoïdes Lacepede in Loch Lomond: Scotland. The Naturee Conservancy: Edinburgh, 233-264.

Schneider J.C., 1972. Dynamics of yellow perch in single species lakes. Res. Dev. Dep. Mich. Dep. Nat. Resour., 184, 47 p. (cité par Treasurer 1980).

Shestopalova G.N., 1978. Reproductive biology of the Iriklinskoye reservoir bream, Abramis brama L.. J. Ichtyol., 884-888.

Souchon Y., 1984. La reproduction du brochet (Esox lucius L., 1758) dans le milieu naturel : revue bibliographique, 1-33. In : Le Brochet. Gestion dans le milieu naturel et élevage. R. Billard Ed., INRA Paris.

Stacey N.E., Cook A.F., Peter R.E., 1979. Ovulatory surge of gonadotropin in golffish, Carassius auratus. Gen. comp. Endocrinol., 37, 246-249.

Statova M.P., 1973. Sexual maturity, reproduction and fecundity. In : Kuchurganskij liman-okhladitel Moldavsky, M.F. Yaroshenko (Ed.). G.R.E.S., 148169 (cité par Moroszewicz 198).

Swift D.R., 1965. Effect of temperature on mortality and rate of development of the eggs of Windermere char (Salvelinus alpinus). J. Fish. Res. Board Can., 2 (4), 913-917.

Thorpe J.E., 1977a. Morphology, physiology, ecology and behaviour of Perca 
fluviatilis L. and Perca flavescens Mitchill. FAO Fisheries synopsis, 113.

Toner E.D. and G.H. Lawler, 1969. Synopsis of biological data on the pike (Esox lucius L.). FAO Fisheries synopsis, 30 (1).

Treasurer J.W., 1980. Some aspects of the reproductive biology and early life history of Perch, Perca fluvialitis $L$. in two aberdeenshire lochs. D.P. Thesis, University of Aberdeen.

Trebaol L., 1982. L'élevage du brocheton de repeuplement: comparaison de trois systèmes de reproduction. DDA - INA, Paris Grignon, 90 p.

Virolyainen M.P., 1940. cité par Treasurer 1980.

Wilkonska H., 1967. Spawning migration of roach (Rutilus rutilus L.) in lake Sniardwy. Rocz. Nauk Roln., sér. H., $3,517-538$. (en polonais).

Wilkonska H. et Zuremska H., 1982. Effect of environmental factors and egg quality on the mortality of spawn in Core- gonus albula (L.) and Coregonus lavaretus (L.). Polsk. Arch. Hydrobiol., 29, 123-158.

Whiteside M.C., Swindoll C.M., Doolittle W.L., 1985. Factors affecting the early life history of yellow perch, Perca flavescens. Env. Biol. Fish. vol. 12, 47-56.

Zawisza J., Bzackiel T., 1970. Gonad development, fecundity and egg survival in Coregonus albula L., 363-397. In C. Lindsey and C. Wood (ed.). Biology of coregonid fishes. University of Manitoba Press. Winnipeg. Manitoba.

Zuremska H., 1967. Mortality estimation of roach (Rutilus rutilus L.) eggs and larvae on lacustrine spawning grounds. Rocz. Nauk. Roln., sér. H, 3, 539-556 (en polonais).

Zuremska., 1982. Egg mortality and its causes in Coregonus albula (L.) and Coregonus lavaretus (L.) in two Masurian lakes. Polsk. Arch. Hydrobiol., 28, 29-70. 